\title{
Estudio descriptivo de la producción caprina tradicional en las regiones mixteca y valles centrales de Oaxaca, México
}

\section{Descriptive study of the traditional goat production in the mixteca and central valleys regions of Oaxaca, Mexico}

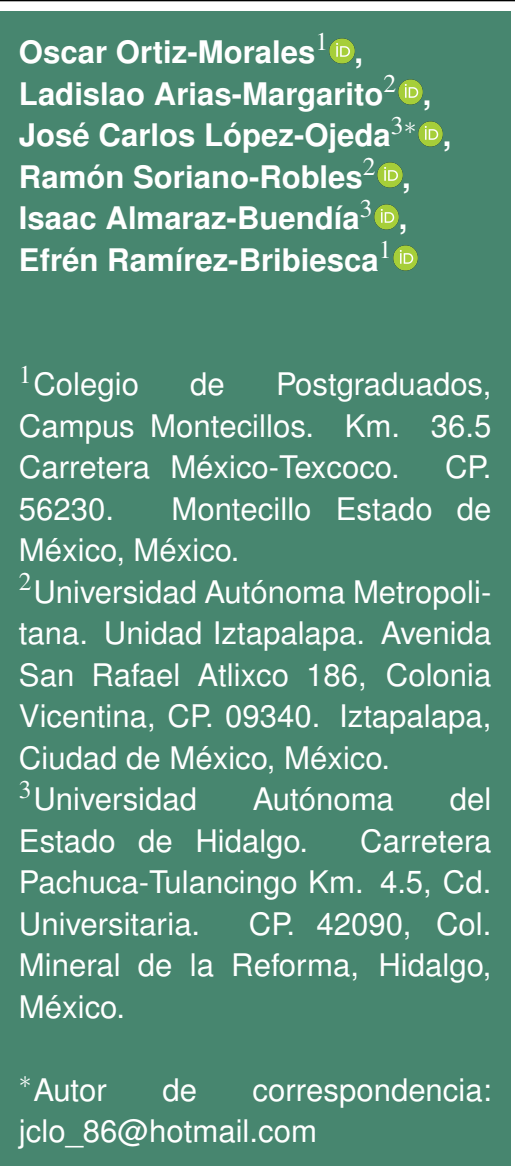

Artículo científico

Recibido: 04 de febrero 2021

Aceptado: 10 de junio 2021

Como citar: Ortiz-Morales O, Arias-Margarito L, López-Ojeda JC, Soriano-Robles R, AlmarazBuendía I, Ramírez-Bribiesca E (2021) Estudio descriptivo de la producción caprina tradicional en las regiones mixteca y valles centrales de Oaxaca, México. Ecosistemas y Recursos Agropecuarios 8(2): e2840. DOI: 10.19136/era.a8n2.2840
RESUMEN. Los conocimientos tradicionales que poseen productores caprinos de Oaxaca se han heredado de generación en generación por casi 500 años. Este estudio describe la producción caprina tradicional con énfasis en su contexto social, ecológico y fenotipos usados en la Mixteca (MX) y Valles Centrales (VC) de Oaxaca. Se utilizó la etnografía para comprender la complejidad de los fenómenos que viven y les son significativos a caprinocultores de estas zonas. El ordenamiento de la información fue sistematizado de acuerdo con un contexto social y cultural, gestión del acervo genético, contexto ecológico e importancia para medios de vida. Como resultados relevantes, el conocimiento caprino es mantenido por personas que rondan 50 años. Existen dos estrategias de producción: trashumante y no trashumante. Se describen cuatro tipos de cabras: Pastoreña, Chinchorrera/Corralera, Fina y Criolla. En VC las cabras se utilizan para la obtención de leche. En la MX el objetivo es la producción de carne. Existen diferentes tipos de pastoreo lo cual indica una clara adaptación de las cabras a condiciones ambientales cambiantes; además, el pastoreo trashumante permite aprovechar la disponibilidad de forraje de acuerdo con la estación del año. Los sistemas de producción caprina tradicional en la MX y VC son heterogéneos; además, coexisten producciones trashumantes y no trashumantes que son clasificadas en actividad primaria o complementaria, respectivamente. Se caracterizan por la mano de obra y tipo de cabra utilizadas, así como el número de animales por hato, el tipo de manejo durante el pastoreo y el objetivo de producción.

Palabras clave: Cabras, características morfo-estructurales, producción, pastoreo, trashumancia.

ABSTRACT. The traditional knowledge of goat farmers in Oaxaca has been inherited from generation to generation for nearly 500 years. This study describes the traditional goat production with emphasis on its social, ecological, and genetic resources in the Mixteca (MX) and Central Valleys (VC) regions of Oaxaca. Ethnography was the method used to assess the complexity of the significant phenomena for goat farmers. The ordering of information was systematized according to a social and cultural context, management of genetic resources, ecological context, and importance for livelihoods. Main results show that goats are handled by 50- year-old farmers. There are two production strategies: transhumant and non-transhumant. Four goat genotypes are described: Pastoreña, Chinchorrera/Corralera, Fina, and Criolla. In VC, goats are exploited mainly for milk production. In the MX, the objective is meat production. Different grazing practices are used, which indicates a clear adaptation of goats to changing environmental conditions. Also, movement of goat herds make use of the availability of forage according to the season of the year. It was concluded that traditional goat production in $\mathrm{MX}$ and $\mathrm{VC}$ is heterogeneous. Besides, transhumant, and non-transhumant productions coexist and are classified as a primary or complementary activity, respectively. They are characterized by the workforce and type of goat used, as well as the number of animals per herd, the type of handling during grazing, and the production target.

Key words: Goats, morpho-structural characteristics, production, grazing, transhumance. 


\section{INTRODUCCIÓN}

México se caracteriza por ser un país importante en la producción de cabras en América Latina, actualmente cuenta con una población estimada de 8.8 millones de animales (SIAP 2020). En Oaxaca, la crianza de cabras junto con la agricultura son las principales actividades productivas y de subsistencia de diversos grupos indígenas (Romero Frizzi 1990, Mendoza García 2002). Particularmente, la producción de caprinos ha generado un amplio conocimiento relacionado con el aprovechamiento y gestión de cabras y recursos naturales (Franco-Guerra et al. 2008, Arias et al. 2011); lo que ha dado como resultado un tipo de cabra conocida como Pastoreña (Domínguez et al. 2018). Estas experiencias son denominadas por Nazarea (2006) como conocimientos ecológicos tradicionales, que han sido generados de manera empírica, mediante ensayo de prueba y error, y transmitidos de generación en generación. La acumulación de estos conocimientos ha contribuido a la construcción de una memoria biocultural que se ha modificado con el tiempo, permitiendo una adaptación continua a condiciones ambientales y sociales cambiantes, generando nuevos conocimientos (Dehouve et al. 2004, Cabrera et al. 2011).

Por otro lado, la migración de los jóvenes a los Estados Unidos de América y la falta de relevo generacional están ocasionando la pérdida paulatina de conocimientos sobre la producción caprina (Varese y Escárcega 2004, Oteros-Rozas et al. 2013). Además, de marginalización, desvalorización o mala interpretación de los sistemas de producción de caprinos, lo que causa desconfianza entre los principales actores de esta actividad y dificulta el trabajo de los investigadores al recabar información (Perezgrovas 2014). En la región Mixteca, hay estudios descriptivos de la producción caprina que han realizado diversos grupos de investigadores (Rodríguez et al. 2011, Ramírez et al. 2014, Soriano et al. 2017). Pero uno de los puntos importantes que falta fortalecer, es la descripción del conocimiento arraigado que tienen los pastores para mantener sus tradiciones de manejo zootécnico como la selección de cabras por color del pelo, características de los cuernos y actividad productiva. Diferentes enfoques metodológicos se han abordado en el estudio del conocimiento ecológico tradicional para su recuperación y revalorización en la producción animal (Alves et al. 2010, Matiuti et al. 2012, Perezgrovas 2014). Uno de estos enfoques es el propuesto en la red LIFE (Local Livestock for Empowerment of Rural People) que se basa en documentar el conocimiento, conceptos y prioridades de comunidades ganaderas en diversas partes del mundo (Sansthan y KöhlerRollerfson 2005). El estado de Oaxaca tiene la mayor población caprina (SIAP 2020), por lo que es necesario indagar en el conocimiento de los productores de cabras en algunas regiones del estado. El presente estudio tiene la hipótesis que en la Mixteca y Valles Centrales del estado de Oaxaca hay diferencias en el manejo zootécnico, comercial y fenotipos en la producción caprina tradicional. Por lo tanto, el objetivo fue describir la producción caprina tradicional con énfasis en su contexto social, ecológico y tipos de cabras utilizadas en la Mixteca y Valles Centrales del estado de Oaxaca.

\section{MATERIALES Y MÉTODOS}

\section{Área de estudio}

El trabajo se desarrolló en 10 localidades de la región Mixteca (MX, 17 48' 00" LN $97^{\circ} 46^{\prime}$ 00" LO) y Valles Centrales (VC, $17^{\circ} 48^{\prime} 00^{\prime \prime}$ LN $97^{\circ} 46^{\prime}$ 00" LO) del estado de Oaxaca (Figura 1) durante el 2017 y 2018. Ambas regiones se ubican en la provincia fisiográfica de la Sierra Madre del Sur, la vegetación corresponde a selva baja caducifolia y bosque de pino-encino. La altitud promedio es de 1862 y 1713 para MX y VC, respectivamente. La temperatura y precipitación media anual en la MX es de $19^{\circ} \mathrm{C}$ y 940 $\mathrm{mm}$, mientras en $\mathrm{VC}$ corresponde a $20^{\circ} \mathrm{C}$ y $558 \mathrm{~mm}$, respectivamente (INEGI 2004).

\section{Compilación de la información y muestreo}

Se utilizó el método etnográfico (Álvarez 2011) para estudiar la complejidad de los fenómenos que viven y les son significativos a las personas involucradas en la producción caprina. A falta de un censo de productores en ambas regiones, se utilizó un 


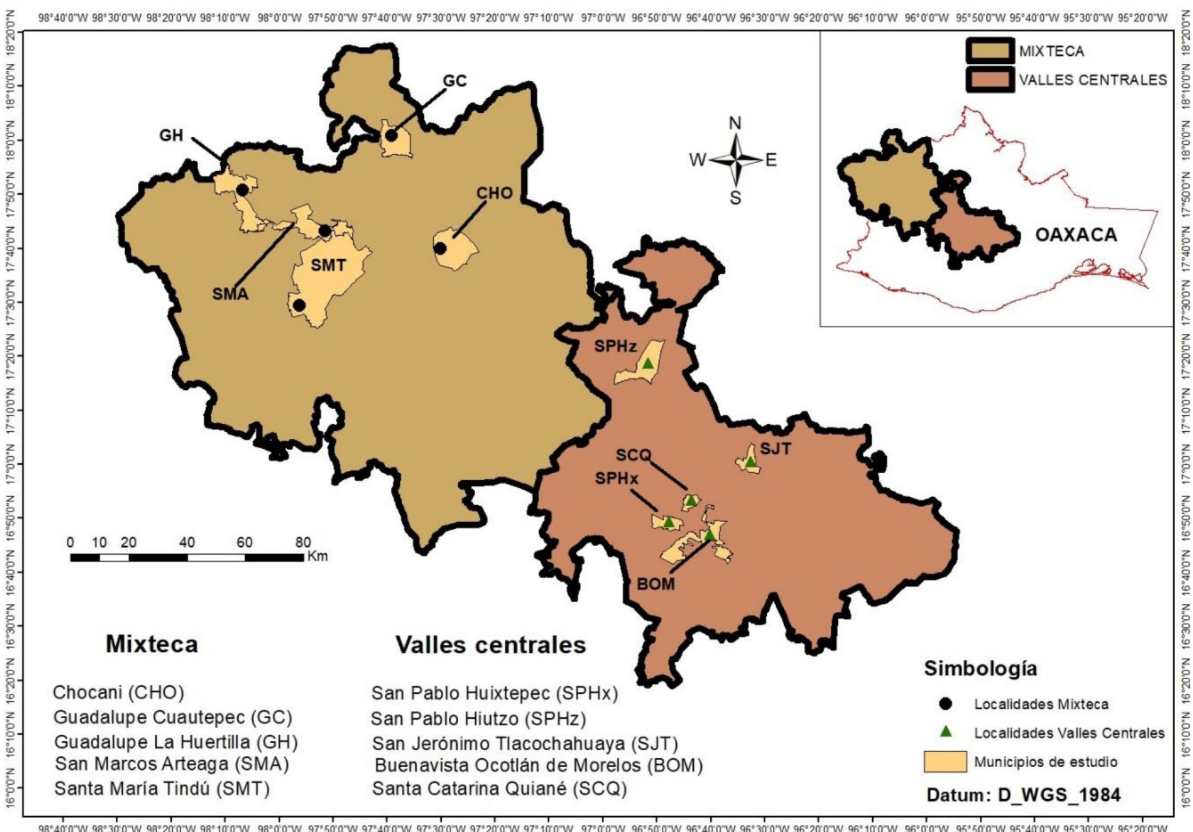

Figura 1. Ubicación de las localidades de estudio en las regiones Mixteca y Valles Centrales, Oaxaca, México.

muestreo no probabilístico, aplicando la metodología bola de nieve (Otzen y Manterola 2017). En ambas regiones, se entrevistó de manera individual (productor) o grupal (productor y familia) a 29 caprinocultores en total, 15 en MX y 14 en VC. Con el propósito de establecer un trato de confianza se convivió con los productores y sus familias para luego acompañarlos en los recorridos cotidianos con sus hatos de cabras, esto ayudó a un mejor flujo de la información. De esta manera se registró edad, experiencia en la actividad caprina, principal actividad económica y laborales complementarias, de cada productor. Además, se recopilaron los conocimientos tradicionales que los productores poseen con respecto a tipos de cabra: color de pelo, forma de cuerno, perfil fronto-nasal, posición de orejas y tipo de ubre; tamaño de hatos, manejo del pastoreo, estacionalidad de especies forrajeras, comercialización, tipo de enfermedades, así como desempeño reproductivo y productivo. Para el tema productivo seis productores por región, permitieron que se pesaran 5 animales en sus hatos en junio durante la época de lluvia, los animales fueron seleccionados por sexo y al nacimiento.
Interpretación y análisis de la información

El ordenamiento de la información fue sistematizado para su análisis descriptivo exploratorio, de acuerdo a cuatro categorías planteados por el método LIFE (Sansthan y Köhler-Rollerfson 2005): contexto social y cultural, gestión del acervo genético, contexto ecológico e importancia para medios de vida.

\section{RESULTADOS}

\section{Contexto social y cultural de los productores}

Los productores tienen una edad promedio de $52 \pm 12$ y $48 \pm 13$ años en MX y VC, respectivamente, con nivel de estudios básicos. La experiencia que tienen en la actividad caprina es amplia, debido a que ellos son la segunda generación dedicados a esta actividad. En la región MX, la cría de caprinos trashumante es una actividad primaria, debido a que es la fuente principal de ingresos del $20 \%$ de los entrevistados. Por otro lado, en MX y VC la cría de caprinos no trashumante se considera como actividad complementaria, debido a que representa una fuente 
de ahorro económico familiar, autoconsumo y se complementa con actividades agrícolas. En cuanto a la mano de obra en la actividad caprina puede ser familiar o asalariada. En la actividad caprina primaria, los dueños de las cabras contratan a pastores para el cuidado de los animales que se mantienen en trashumancia. Los pastores reciben un pago de $\$ 3500$ (199 USD) y $150 \mathrm{~kg}$ de maíz al mes. Este último pago se debe a que los pastores se dedican exclusivamente al cuidado y pastoreo de las cabras, además viven junto con el ganado en el monte. Los pastores viajan con sus familias, formando la unidad social básica conocida como rancho, refugio construido con lonas a manera de carpa. Los ranchos son armados y desarmados con el cambio de zona de pastoreo. En la actividad caprina complementaria, la mano de obra es familiar; las esposas, niños, yernos, nueras o adultos mayores se encargan del cuidado y pastoreo de las cabras, sin obtener remuneración por su trabajo. Las labores principales son la limpieza de corrales y la conducción de las cabras al pastoreo, estas actividades se rolan entre los familiares dependiendo del tiempo que tengan disponible o si se los permite la actividad adicional que realiza cada uno. Por lo regular, las cabras se alojan en los solares, a un costado de la casa de los productores. En los últimos cinco años, ningún productor afirmó pertenecer a alguna asociación de productores o recibir apoyos en especie o económica de alguna dependencia de gobierno. No obstante, los productores jubilados mencionan que han existido programas gubernamentales que han introducido razas especializadas como Boer o Nubia, para mejorar la productividad, pero estos animales tienen problemas de adaptación, son enfermizos o no están adaptados a las jornadas de pastoreo.

\section{Gestión del acervo genético. Tipos de cabras}

En la región $\mathrm{MX}$, la producción caprina tiene como objetivo la ceba de animales que se destinan a la barbacoa o la matanza, realizada en Tehuacán, Puebla y Huajuapan de León, Oaxaca. En VC, el enfoque principal de los caprinocultores es la obtención de leche, para la elaboración de quesos. La diferencia entre las producciones de ambas regiones ha hecho que exista una amplia diversidad respecto a los fenotipos de cabras. La actividad caprina se realiza con cabras denominadas por los mismos productores como Criollas (Figura 2). Las cuales presentan características morfo-estructurales que han sido mantenidas por los productores (Tabla 1). En la MX, Pastoreña y Chinchorrera/Corralera son las denominaciones que dan los caprinocultores a los tipos de cabra que utilizan. Las cabras Pastoreñas son predominantemente blancas y crema, aunque existen colores recesivos como el café o negro. Este tipo de ganado es común en las comunidades de San Marcos Arteaga, Guadalupe Cuautepec y Santa María Tindú. Por otro lado, la cabra Chinchorrera/Corralera, posee una amplia gama de tonalidades en su pelo: negro, blanco, café, colorada y una combinación de estos colores. Este ganado es predominante en las comunidades de Guadalupe la Huertilla y Chocani. Las cabras Pastoreñas presentan ubres globosas y ovales; pero esta última no es del agrado en los productores, pues sufren lesiones constantes durante el pastoreo y se dificulta el amamantamiento de las crías. En VC, Finas y Criollas son las denominaciones que dan los productores a las cabras que utilizan, los hatos de cabras Finas se encuentran en San Pablo Huixtepec y San Jerónimo Tlacochahuaya, en las demás comunidades los hatos mantienen una mezcla de Finas y Criollas. Los animales de pelo colorado, café y negra son preponderantes en ambos tipos de cabras. La posición de orejas y el tipo de ubre son características predominantes que los productores eligen para distinguir cabras Finas de Criollas.

\section{Tamaño de hatos}

En VC, los hatos de cabras Finas o Criollas tienen una densidad máxima de 60 animales, y en ocasiones se acompañan de vacas y borregos. En MX, los hatos de cabras Chinchorreras/Corraleras tienen una densidad de 150 animales; en hatos de cabras Pastoreñas, la densidad puede variar desde 300 hasta 1000 animales. Cuando la actividad caprina es primaria (trashumante), un solo pastor cuida hasta 500 animales; y sí la cantidad es mayor, será necesario contratar a otro pastor, pero los animales se mantienen formando un solo rebaño. 


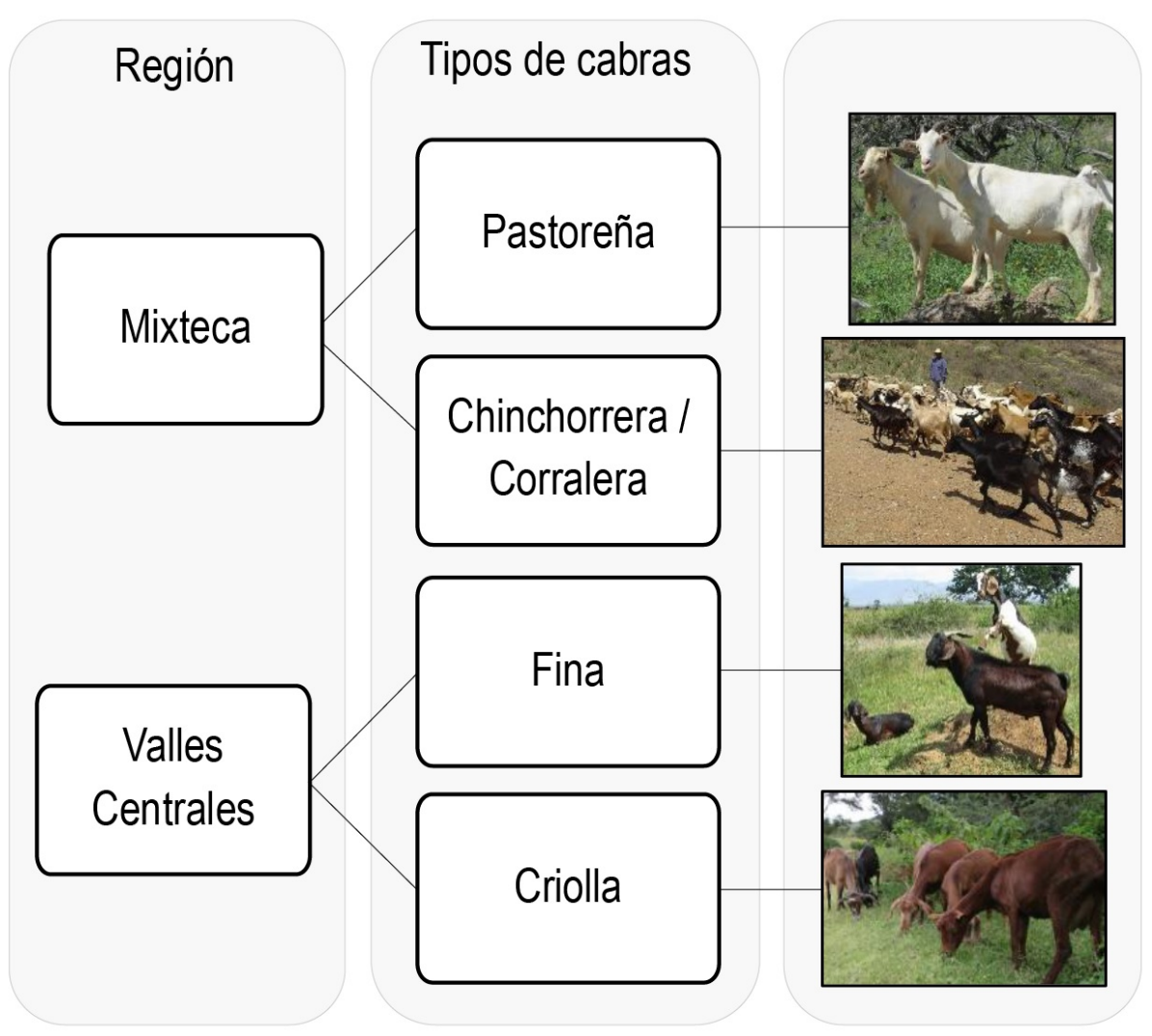

Figura 2. Principales fenotipos de cabras en la región Mixteca y Valles Centrales, Oaxaca.

Tabla 1. Caracteres morfo-estructurales en caprinos de la región Mixteca y Valles Centrales de Oaxaca.

\begin{tabular}{|c|c|c|c|c|}
\hline \multirow[b]{2}{*}{ Tipo de cabra } & \multicolumn{2}{|r|}{ Mixteca } & \multicolumn{2}{|c|}{ Valles Centrales } \\
\hline & Pastoreña & Chinchorrera/Corralera & Fina & Criolla \\
\hline Color de pelo & -Blanca, crema & -Negra, blanca, café, colorada & \multirow{2}{*}{\multicolumn{2}{|c|}{$\begin{array}{l}\text {-Colorada, negra, café, blanco } \\
\text {-Hembras: CAPa, CAPA, CAPar, AC }\end{array}$}} \\
\hline \multirow[t]{2}{*}{ Forma de cuernos } & \multicolumn{2}{|c|}{-Hembras: CAPA, CAPa, CAPar, AC } & & \\
\hline & -Machos: EPD y AC & -Machos: EPD, PPD, AC & \multicolumn{2}{|c|}{-Machos: PPD y AC } \\
\hline Perfil fronto-nasal & -Recto y cóncavo & -Recto, cóncavo y convexo & -Recto & -Recto \\
\hline Posición de orejas & $-\mathrm{RH}$ & $-\mathrm{RH}, \mathrm{SP}, \mathrm{P}$ & $-P$ & $-R H$ y $S P$ \\
\hline Tipo de ubre & -Globosa y oval & -Globosa, periforme, oval & -Oval, periforme & -Globosa y periforme \\
\hline
\end{tabular}

El pastoreo de estos animales se practica en sitios de abundante vegetación que regularmente están alejados de los pueblos, además estos sitios son exclusivos, es decir no se comparten con otros rebaños. La venta de animales a la matanza durante los meses de octubre y diciembre ocasiona una disminución de cabras en los rebaños; de este modo, se reduce la presión de pastoreo que ejerce el ganado durante la época de sequía y asegura la sobreviven- cia del rebaño sobrante. Cuando la actividad caprina es complementaria (no trashumante), los productores afirman que la densidad de población en los rebaños no debe sobrepasar los 150 animales. Debido a que durante la época de sequía escasea el forraje y existe mayor competencia por los sitios de pastoreo que comparten con otros productores, por lo que es necesario complementar con residuos agrícolas. 


\section{Instalaciones}

Los corrales de Chichorreras, Finas y Criollas se usan para alojar a las cabras cuando no están pastoreando y se localizan al costado de la vivienda del productor. El $80 \%$ de estos corrales tienen techo y están construidos con malla metálica ciclónica o materiales de la región, como el tallo seco de la inflorescencia de maguey o de cactus en la MX y de carrizo en VC. En contraste, el $60 \%$ de corrales no mantienen un declive natural para favorecer el escurrimiento de desechos, y el 70\% tienen comederos o bebederos rústicos. En general, el espacio por animal es de $1 \mathrm{~m}^{2}$. Para la cría de cabras Pastoreñas no se usan corrales, los productores comentan que es insostenible utilizar corrales durante la trashumancia y que les resultaría muy costoso por el número de animales; sin embargo, improvisan corrales de manejo para desparasitar e inmunizar a los animales, estos corrales son itinerantes y están hechos de malla metálica. El agua que utiliza el ganado Pastoreño se obtiene de manantiales tanto en época de sequía como de lluvia. Mientras que, para las demás cabras se consigue de la toma de abastecimiento del hogar en época de sequía y de riachuelos en época de lluvia.

\section{Enfermedades}

En ambas regiones, cada 6 meses se desparasita y vitamina a las cabras. Las enfermedades más comunes son conjuntivitis, timpanismo, linfadenitis caseosa, paratuberculosis, pasteurelosis (posible Mannheimia haemolytica), gabarro (posible Fusobacterium necrophorum), ectima contagioso y derriengue (Lyssavirus). Ocasionalmente se inmuniza contra pasteurelosis y clostridiasis con bacterinas múltiples que les sugieren los veterinarios de la región. Pero no se tiene un manejo adecuado de estas enfermedades por el desconocimiento de los productores, y la falta de asistencia técnica.

\section{Contexto ecológico. Pastoreo}

El pastoreo del ganado se realiza en tierras comunales en la MX y en tierras ejidales y particulares en VC. El tiempo dedicado a la actividad es de 8 $\mathrm{h}$ al día, aproximadamente. En producciones de ganado Chinchorrero, Fino y Criollo, el pastoreo comienza entre las 10:00 y 11:00 am; en la época de lluvia es común que los animales pastoreen en la periferia de las comunidades, conocida como monte, aprovechando la biomasa de diversas especies arbóreas, arbustivas y herbáceas; mientras que en la época de sequía es usual pastorear entre áreas agrícolas y monte. Ocasionalmente, algunos productores complementan la alimentación del ganado con residuos agrícolas de maíz, frijol o cacahuate. La alimentación de cabras Pastoreñas ocurre al transitar en el monte, en partes altas en la época de sequía y en partes bajas en la de lluvia. Las áreas que pastorean son utilizadas por una semana y regresan hasta un año después. El pastoreo se realiza en dos periodos conocidos como almuerzo y cena, el almuerzo es de 08:00-12:00 h; después el ganado regresa al rancho para sestear (descansar) durante 3 h; y la cena se efectúa de 15:00-19:00 h. Para el pastoreo de estos animales, es necesario que los productores renten extensiones de monte, el costo asciende a \$5 000 pesos al año (285 USD); no obstante, el pago es variable dependiendo del tamaño del rebaño, el área rentada y el tiempo de permanencia. En ambas regiones es común que cada 15 días se proporcione sal a las cabras.

\section{Calendario estacional de forrajes}

En ambas regiones, el forraje disponible varía a través del año, lo que ocasiona un ciclo de pérdidaganancia de peso en las cabras. Los caprinocultores mencionan que las principales especies consumidas por cabras en el pastoreo son: en VC, en época de lluvia, Mimosa texana, M. pudica, Acacia pennatula, Eysenhardtia polystachya, Simsia calva, Melampodium divaricatum; en época de sequía, Leucaena leucocephala, Rhynchelytrum repens, Bouteloua curtipendula y Liria psittacanthus. En MX durante la época de lluvia, E. polystachya, Dalea gregii, Bursera sp., A. cochliacatha, Montanoa sp., A. farnesiana; en época de sequía, A. bilimekii, Tecoma stans, Dodonea viscosa, Lysiloma acapulcense, Quercus sp. y Rhus oaxacana. Además, las bellotas de Quercus sp. y las vainas de Prosopis laevigata, $A$. pennatula, $L$. leucocephala, $A$. farnesiana y $A$. 
cochliacatha son valoradas por los productores durante la época de sequía. También se reporta la intoxicación de cabras por el consumo de Ipomea murucoides, Ipomea pauciflora, Karwinskia humboldtiana, Tribulus terrestres y Datura stramonium.

\section{Importancia para los medios de vida. Desempeño reproductivo y productivo}

En ambas regiones, los productores no llevan registro de indicadores productivos o reproductivos del rebaño. En la Tabla 2 se muestran los pesos corporales promedio de cabras Pastoreña, Chinchorrera, Fina y Criolla, al nacimiento y por sexo. Respecto al ámbito reproductivo, la fecundación de las cabras no es controlada y difiere entre regiones y tipos de cabra (Tabla 3). En la MX se mantiene una relación hembra:macho de 40:1, mientras que en VC es 20:1. En ganado Fino y Criollo, frecuentemente los sementales son rentados, por sus características fenotípicas deseables (orejones), hasta en $\$ 200$ (10.7 USD) por día. En producciones de cabras Chinchorreras, Finas y Criollas esporádicamente cuentan con áreas de maternidad para separar y proteger a los cabritos neonatos. En producciones de cabras Pastoreñas, los pastores eligen sitios de maternidad y los llaman ahijaderos, regularmente con presencia de arbustos de $A$. cochliacantha, pues aportan sombra y sus troncos sirven para sujetar a los cabritos enseguida de su nacimiento, para que no se extravíen mientras las madres pastorean. Se rasura la cola de la madre y cría después de la parición para prevenir infecciones y evitar que se pegue el meconio en la región perianal de la cría. Además, los pastores ponen una marca única e idéntica con el corte de pelo en la costilla derecha de la madre y cría, la cual se le conoce como ahijar. En VC, el destete de las crías ocurre a los dos meses, mientras que en la MX no se realiza. En ambas regiones, la mayor tasa de mortalidad de cabritos se presenta en la época de sequía.

\section{Comercialización}

En la MX, el ganado Pastoreño tiene los mejores precios de venta, una cabra de 4 o 5 años pude venderse hasta en $\$ 1100$ pesos (59 USD); mientras que el ganado Chinchorrero oscila entre los
$\$ 500$ (27 USD) y $\$ 900$ pesos (48 USD). La mayor actividad comercial de ganado caprino se realiza entre octubre y noviembre, durante la temporada de matanza que se realiza en Tehuacán, Puebla y Huajuapan de León, Oaxaca. En la matanza el producto principal es la cadera de cabras, cada pieza tiene un costo de $\$ 770$ (41 USD). También se obtiene chito, carne seca salada $\left(\$ 300 \mathrm{~kg}^{-1}\right)$ (16 USD), costillas fritas $\left(\$ 300 \mathrm{~kg}^{-1}\right.$ ) (16 USD) y chicharrón de ubre $\left(\$ 550 \mathrm{~kg}^{-1}\right.$ ) (29 USD). Por otro lado, los cabritos de dos meses de edad son vendidos entre $\$ 400$ (21 USD) y $\$ 500$ pesos (27 USD). Algunos productores Pastoreños venden sementales o hembras a productores chinchorreros. En VC, los animales se venden en los baratillos, tianguis establecidos para la venta de especies ganaderas. Los cabritos de dos a tres meses de edad son vendidos entre \$500 (27 USD) y $\$ 600$ pesos (32 USD). Las hembras mayores de 5 años son engordadas para alcanzar precios de venta entre $\$ 1600$ y $\$ 1800$ pesos (86-97 USD) o vendidas como gestantes hasta en $\$ 2000$ pesos (108 USD). En todas las comunidades de VC los machos para venta son castrados para disminuir su olor, los cuales se venden hasta en $\$ 2500$ pesos (135 USD). La leche tiene un precio entre $\$ 8$ (0.43 USD) y $\$ 10$ pesos (0.54 USD) por litro, toda la producción se utiliza para la elaboración de queso fresco. Con un litro de leche se elaboran un queso de $150 \mathrm{~g}$ y con $2 \mathrm{~L}$ uno de $250 \mathrm{~g}$, con precio entre $\$ 25$ (1.35 USD) y $\$ 45$ pesos (2.43 USD), respectivamente. Las cabras son ordeñadas después del destete de sus crías que se realiza a los dos meses de edad.

\section{DISCUSIÓN}

\section{Contexto social y cultural de los productores}

En referencia a la experiencia de los productores, Trujillo Ortiz et al. (2010) indican que el promedio de experiencia en la actividad caprina es de 27 años, y la razón principal de esta actividad es por tradición y la ven como una fuente de empleo. Mientras que Escareño Sánchez et al. (2011) mencionan que productores del norte del país tienen experiencia de 1 a 9 años en cabras lecheras en sistemas extensivos. Pero es común que las mujeres, niños y 
Tabla 2. Valores medios \pm desviación estándar de pesos corporales en caprinos de la región Mixteca y Valles Centrales de Oaxaca.

\begin{tabular}{lcccc}
\hline Peso corporal $(\mathrm{kg})$ & Pastoreña & Chinchorrera & Fina & Criolla \\
\hline Al nacimiento & $2.9 \pm 0.6$ & $2.2 \pm 0.5$ & $2.8 \pm 0.4$ & $2.1 \pm .3$ \\
Hembras & $44.5 \pm 8$ & $38.1 \pm 3$ & $42 . \pm 8$ & $35 \pm 2.8$ \\
Machos & $67.3 \pm 8$ & $56.7 \pm 4$ & $55.6 \pm 3$ & $45.2 \pm 7$ \\
\hline
\end{tabular}

Tabla 3. Estacionalidad de la producción forrajera y ciclo reproductivo en cabras de la región Mixteca y Valles Centrales.

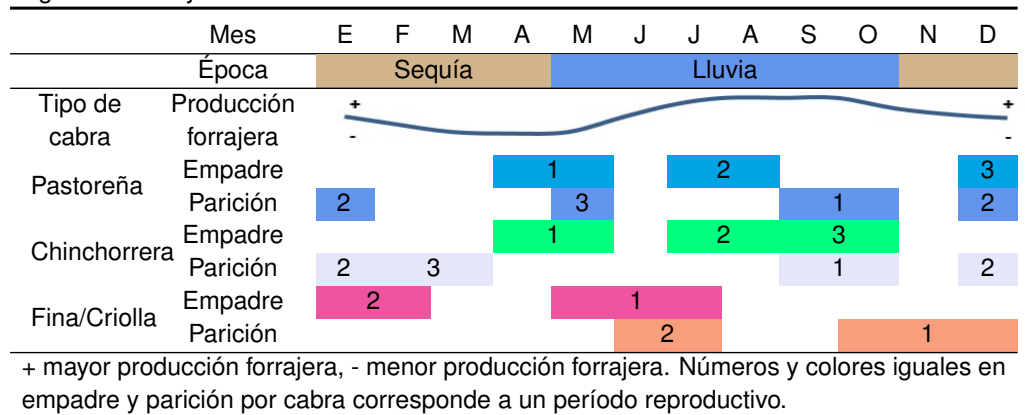

ancianos jueguen un papel fundamental en la actividad caprina (Hernández et al. 2013), ya que ellos se encargan de tareas como limpiar el corral o pastorear a los animales dentro de la producción. En tanto que otro estudio indica que la mano de obra utilizada en los sistemas caprinos es $76 \%$ familiar, donde la mujer aporta alrededor del $15 \%$ y los hijos muestran mayor integración a esta actividad (Valerio et al. 2009). Particularmente, en las regiones del estudio, la fragmentación en la estructura familiar por la alta migración ocasiona que mujeres, niños y ancianos jueguen un papel fundamental en la actividad caprina (Hernández 2011). A pesar de la importancia económica, social y cultural del ganado caprino en ambas regiones son limitados los estudios que describen estos sistemas de producción.

\section{Gestión del acervo genético. Tipos de cabras}

En MX, las cabras Pastoreñas y Chinchorreras descritas coinciden con lo reportado por otros autores (Franco-Guerra et al. 2008, Arias et al. 2011). Este tipo de animales no son exclusivos del estado de Oaxaca, pero sí de la región MX que está integrada por los estados de Puebla (Zepeda et al. 2002) y Guerrero (Cabrera et al. 2011). La similitud de sistemas de producción caprina entre estados, se remonta a la época colonial, debido a que la actividad caprina estaba ampliamente distribuida en la región (Romero Frizzi 1990, Dehouve et al. 2004). En VC, Fuentes et al. (2013) describen la zoometría de caprinos; sin embargo, no distinguen entre cabras Finas y Criollas.

\section{Tamaño de hato e instalaciones}

El tamaño del hato está en función de la disponibilidad de alimento para las cabras y sí la producción caprina es una actividad económica primaria - complementaria (Mendoza-Jiménez y OrtegaSánchez 2009, Arias et al. 2011, Franco-Guerra et al. 2014). La ausencia de corrales es común es sistemas trashumantes (Martínez et al. 2014). El hacinamiento de los animales en los corrales es frecuente debido a que difícilmente se exceda el metro cuadrado por animal (García Bonilla et al. 2018).

\section{Enfermedades}

La sanidad es un factor que incide de forma negativa en la productividad de los hatos caprinos y para contrarrestar este efecto no se ha explorado la capacidad de resistencia a las enfermedades de las cabras Criollas (García Bonilla et al. 2018). En ambas regiones hacen falta estudios de diagnósticos que confirmen el agente causal de las patologías, algunos reportes aislados mencionan la presencia de 
brucelosis y derriengue, pero no hay publicaciones al respecto. Pero se sabe que la paratuberculosis caprina está ampliamente diseminada en las principales regiones caprinas (Mixteca y Libres) del Estado de Puebla (Favila et al. 2017). La desparasitación gastrointestinal semestral; la vacunación contra pasteurelosis, clostridiasis y brucelosis, son tareas de medicina preventiva que se practican en los hatos de la MX y VC, como lo reportado en otras regiones del país (Alva-Pérez et al. 2019). En México no se ha extendido la práctica de vacunar contra ectima contagioso, quizás por esta razón no existen productos comerciales en el mercado (Tórtora 1987). En cabras es frecuente la muerte de neonatos por abortos o por síndrome de inanición, principalmente en las cabras primíparas (Romero-Ramírez et al. 1998, López et al. 2012). La presencia de asistencia técnica en hatos ovinos ha demostrado disminuir mortalidad en neonatos y aumentar la tasa de pariciones (MartínezGonzález et al. 2011). La ausencia de asistencia técnica puede causar un mal uso de medicamentos (Torres-Acosta et al. 2003, Alva-Pérez et al. 2019).

\section{Contexto ecológico. Pastoreo}

El tiempo de alimentación de cabras Chinchorreras/Corraleras, Finas y Criollas es semejante a lo descrito por Arias et al. (2011) y Rebollar et al. (2012). Mientras que las particularidades en la alimentación de cabras Pastoreñas coinciden con lo reportado por Martínez et al. (2014). Además, el pastoreo caprino trashumante aprovecha la diversidad vegetal que existe en territorio montañoso (Franco-Guerra et al. 2008); condición fisiográfica que limita el pastoreo de otros tipos de ganado (Mallén 2006).

\section{Calendario estacional de forrajes}

Baraza et al. (2008) afirman que las cabras pierden $4.5 \mathrm{~kg}$ de peso entre enero y mayo a consecuencia de la disminución de la producción de forraje durante la época seca. En lo referente a la composición química de las especies vegetales consumidas por las cabras Chinchorreras, Arias et al. (2014) reportaron que el forraje y las vainas de especies como $A$. cochliacatha, $A$. farnesiana y $P$. laevi- gata tienen un contenido de proteína superior al 15 y $10 \%$, respectivamente. Mientras que Franco-Guerra et al. $(2008,2014)$ identificaron a $E$. polystachya y $A$. pennatula como las especies de mayor preferencia y consumo por cabras Pastoreñas. Además, demostraron que el consumo de forraje es difícil de estimar en condiciones de pastoreo debido la frecuencia de alimentación y a la diversidad de especies de las que se alimentan las cabras. En tanto que MilaArango et al. (2014) describieron los componentes secundarios de I. murucoides e I. pauciflora, señalados como causantes de intoxicación en cabras.

\section{Importancia para los medios de vida. Desempeño productivo y reproductivo}

La ausencia de registros con indicadores reproductivos y productivos en la caprinocultura es común. Los pesos corporales de cabras reportados en esta investigación son semejantes a los mencionados por Alejandre-Ortiz et al. (2016) para cabras criollas; mientras que Villarreal-Arellano et al. (2018) reportan pesos corporales de cabras Pastoreñas al nacimiento, hembras y machos adultos similares. Por otro lado, los periodos de parición reportados para cabras Pastoreñas y Finas/ Criollas coinciden con los periodos señalados por Sierra et al. (1997) y Fuentes et al. (2013), respectivamente. En cuanto a la proporción hembra:macho (50:1) ha sido reportada en sistemas no trashumantes (Mellado 2008) y trashumantes (Martínez et al. 2014) pero puede disminuir, sí la actividad caprina se combina con actividades agrícolas (García Bonilla et al. 2018). Por otro lado, se han descrito distintos ahijaderos (Lanz 2020, Martínez et al. 2014) con el objetivo de asegurar una mayor sobrevivencia de cabritos.

\section{Comercialización}

En MX, la matanza es una actividad que da empleo y aporta a la economía familiar de manera directa o indirecta (Rodríguez et al. 2011, García y Ferreiro 2008). Sin embargo, se debería abordar a fondo esta cadena de comercialización para mejorar los precios de venta a productores caprinos. En VC, la venta de queso contribuye a la economía familiar de caprinocultores. La cercanía con la capital de 
Oaxaca hace que esta actividad pueda ampliar su canal de comercialización.

\section{CONCLUSIONES}

La producción caprina tradicional en la Mixteca y Valles Centrales es heterogénea. Coexisten producciones trashumantes y no trashumantes clasificadas como actividad primaria o complementaria, respectivamente. Se caracterizan por la mano de obra y tipo de cabra utilizadas, así como del número de animales por hato, el tipo de manejo durante el pastoreo y el objetivo de producción. Las características morfo-estructurales de las cabras difiere entre regiones y dependen del objetivo de la producción, así como del tipo de pastoreo. El conocimiento que tienen los productores de la vegetación y del ambiente es amplio, pudiendo identificar las especies consumidas por las cabras durante la época de lluvia y sequía, así como las que son tóxicas. También identifican las mejores áreas de pastoreo con el cambio de estación. El pastoreo trashumante, así como la producción de queso y la elaboración de mole de caderas son un distintivo cultural entre ambas regiones.

\section{LITERATURA CITADA}

Alejandre-Ortiz ME, Rubio-Tabárez E, Pérez-Eguía E, Zaragoza-Martínez L, Rodríguez-Galván G (2016) Los recursos caprinos de México. En: Vargas BJE, Zaragoza ML, Delgado BJV, Rodríguez GG (ed). Biodiversidad caprina iberoamericana. Primera edición. Bogotá, Colombia. 244p.

Alva-Pérez J, López-Corona, LE, Zapata-Campos CC, Vázquez-Villanueva J, Barrios-García HB (2019) Condiciones productivas y zoosanitarias de la producción caprina en el Altiplano de Tamaulipas, México. Interciencia 44: 152-158.

Álvarez RM (2011) La investigación etnográfica: una propuesta metodológica para trabajo social. Trabajo Social 20: 73-97.

Alves AGC, Pires DAF, Ribeiro MN (2010) Conhecimento local e produção animal: uma perspectiva baseada na etnozootecnia. Archivos de Zootecnia 59: 45-56.

Arias L, Soriano R, Sánchez E, González E, Rivera L (2011) Características técnicas y socioeconómicas de los sistemas de producción caprina en un municipio de la Mixteca Baja oaxaqueña. En: Cavallotti VB, Ramírez VB, Martínez CFE, Marcof ÁCF, Cesín VA (ed). La ganadería ante el agotamiento de los paradigmas dominantes. Vol 2. Chapingo, México. pp: 335-345.

Arias L, Soriano-Robles R, Gonzalez-Esquivel CE, Sanchez E (2014) Chemical composition and in vitro digestibility of fodder trees and shrubs consumed by goats in the low Mixteca region of Oaxaca, Mexico. Research Journal of Biological Sciences 9: 92-97.

Baraza E, Ángeles S, García Á, Valiente-Banuet A (2008) New natural resources as diet supplement for domestic goats during the dry season, in the Tehuacán Valley, Mexico. Interciencia 33: 891-896.

Cabrera R, Vargas S, Bustamante Á, Olvera J (2011) Experiencias en la producción de ganado caprino en el estado de Guerrero. Primera edición. Colegio de Postgraduados-Altres Costa-Amic Editores. Puebla, México. 184p.

Dehouve D, Cervantes R, Hvilshoj U (2004) La vida volante. Pastoreo trashumante en la Sierra Madre del Sur, ayer y hoy. Primera edición. Jorale / Universidad Autónoma de Guerrero Editores. México. 135p.

Domínguez MÁ, De la Rosa JDP, Landi V, De la Rosa JP, Vazquez N, Martínez MA, Fuentes-Mascorro G (2018) Genetic diversity and population structure analysis of the Mexican Pastoreña Goat. Small Ruminant Research 168: 76-81. 
Escareño Sánchez LM, Wurzinger M, Pastor LF, Salinas H, Sölkner J, Iñiguez L (2011) La cabra y los sistemas de producción caprina de los pequeños productores de la Comarca Lagunera, en el norte de México. Revista Chapingo Serie Ciencias Forestales y del Ambiente 17: 235-246.

Favila C, López DC, Morales RJ, Aparicio D (2017) Situación epidemiológica de la paratuberculosis en las principales regiones caprinas del estado de Puebla, México. Quehacer Científico de Chiapas 12: 36-45.

Franco-Guerra FJ, Sánchez-Rodríguez M, Hernández HJ, Espino-Barros O, Camacho RJ, Hernández RM (2008) Evolución del comportamiento alimentario de cabras criollas en especies arbóreas y arbustivas durante el pastoreo trashumante, México. Zootecnia Tropical 26: 383-386.

Franco-Guerra FJ, Sánchez RM, Camacho RJC, Hernández HJE, Villarreal OA, Rodríguez EL, Marcito O (2014) Consumo de especies arbóreas, arbustivas y sus frutos y herbáceas por cabras en pastoreo trashumante en la Mixteca Oaxaqueña, México. Tropical and Subtropical Agroecosystems 17: 267-270.

Fuentes G, Martínez JMS, Alejandre OM, Chirinos Z, Ricardi CLC (2013) Zoometría y distribución de partos de la cabra criolla de los Valles Centrales de Oaxaca. Actas Iberoamericanas de Conservacion Animal 3: 150-154.

García Bonilla DV, Vargas López S, Bustamante González Á, Torres Hernández G, Calderón Sánchez F, Olvera Hernández JI (2018) La producción de caprinos para carne en la montaña de Guerrero, México. Agricultura Sociedad y Desarrollo 15: 1-17.

García Chacon D, Ferreiro Ontiveros GM (2008) La matanza caprina en la Mixteca Oaxaqueña. Gaceta del Instituto del Patrimonio Cultural del Estado de Oaxaca 15: 19-28.

Hernández JE, Franco FJ, Villarreal OA, Camacho JC, Pedraza RM (2013) Caracterización socioeconómica y productiva de unidades caprinas familiares en la Mixteca Poblana. Archivos de Zootecnia 60: 175-182.

INEGI (2004) Síntesis de información geográfica del estado de Oaxaca. Instituto Nacional de Estadística, Geografía e Informática. Primera edición. México. 180p.

Lanz B (2020) La pesca del cabrito. En: Cardenas JR (ed). La senda del cabrito. Segunda edición. Ediciones Larousse. Ciudad de México, México. pp: 43-47.

López G, Díaz VLD, Serrano BA, Muñoz ML, Cartagena TM, Ramírez CMR (2012) Niveles sanguíneos de cortisol y receptores a glucocorticoides en hígado y placenta de cabras en diferentes etapas de la gestación. Veterinaria México 43: 213-223.

Mallén C (2006) Diagnostico Forestal y Ambiental del estado de Oaxaca. International Tropical Timber Organization. http://www.itto.int/files/itto_project_db_input/2576/Technical/DIAGNOSTICO\%20AMBIENTAL\%20Y \%20FORESTAL\%20DEL\%20ESTADO\%20DE\%20OAXACA.pdf. Fecha de consulta: 13 de enero de 2021.

Martínez-González EG, Muñoz-Rodríguez M, García-Muñiz JG, Santoyo-Cortés VH, Altamirano-Cárdenas JR, Romero-Márquez C (2011) El fomento de la ovinocultura familiar en México mediante subsidios en activos: lecciones aprendidas. Agronomía Mesoamericana 22: 367-377.

Martínez RD, Torres G, Martínez S (2014) Caracterización fenotípica, productiva y reproductiva de la cabra blanca Criolla del "Filo Mayor" de la Sierra Madre del Sur en el estado de Guerrero. Nova Scientia 6: 25-44.

Matiuti M, Bogdan AT, Matiuti CL (2012) The ethno-zootechnical solution for preservation and development of zoogenetic heritage in Romania and Europe. Bulletin UASVM of Agricultural Science and Biotechnologies 69: 136-143.

Mellado M (2008) Técnicas para el manejo reproductivo de las cabras en agostotadero. Tropical and Subtropical Agroecosystems 9: 47-63. 
Mendoza-Jiménez A, Ortega-Sánchez JL (2009) Caracterización de la caprinocultura en el municipio de Tepelmeme Villa de Morelos, Oaxaca, México. Revista Chapingo Serie Zonas Áridas 8: 75-80.

Mendoza García E (2002) El ganado comunal en la Mixteca alta: de la época colonial al siglo XX. El caso de Tepelmeme. Historia Mexicana 51: 749-785.

Mila-Arango R, Ramírez-Bribiesca E, Soto-Hernández R, Hernández-Mendo O, Torres-Hernández G, MelladoBosque M (2014) Identificación y estudio fitoquímico de dos especies de cazahuate en la intoxicación de cabras en una comunidad de la Mixteca Oaxaqueña. Agricultura Sociedad y Desarrollo 11: 463-479.

Nazarea VD (2006) Local knowledge and memory in biodiversity conservation. Annual Review of Anthropology 35: 317-335.

Oteros-Rozas E, Ontillera-Sánchez R, Sanosa P, Gómez-Baggethun E, Reyes-García V, González JA (2013) Traditional ecological knowledge among transhumant pastoralists in Mediterranean Spain. Ecology and Society 18: 33-51.

Otzen T, Manterola C (2017) Técnicas de muestreo sobre una población a estudio. International Journal of Morphology 35: 227-232.

Perezgrovas R (2014) Antología sobre etnoveterinaria. Origen y evolución en Chiapas. Primera edición. Instituto de Estudios Indígenas- Universidad Autónoma de Chiapas. Chiapas, México. 398p.

Ramírez JMP, Sánchez OM, Ortiz BR, Zaragoza RJL, Ricardi DLC, Fuentes-Mascorro G (2014) Sistema se producción y zoometría de la cabra pastoreña. Acta Iberoamericana de Conservación Animal 4: 231-233.

Rebollar S, Hernández J, Rojo R, Guzmán E (2012) Gastos e ingresos en la actividad caprina extensiva en México. Agronomía Mesoamericana 23: 159-165.

Rodríguez LG, Gamboa AJG, García SJA, Rivera MJG (2011) Tradición sociocultural de la caprinocultura en el estado de Oaxaca: análisis sobre el potencial económico en beneficio de la región de Huajuapan de León. En: Cavallotti VB, Ramírez VB, Martínez CFE, Marcof ÁCF, Cesín VA (ed). La ganadería ante el agotamiento de los paradigmas dominantes. Vol 2. Chapingo, México. pp: 335-345.

Romero RCM, López G, Luna MM (1998) Abortion in goats associated with increased maternal cortisol. Small Ruminant Research 30: 7-12.

Romero Frizzi MÁ (1990) Economía y vida de los españoles en la Mixteca Alta: 1519-1720. Primera edición. Colección Regiones de México. Instituto Nacional de Antropología e Historia. México. 636p.

Sansthan LPP, Köhler-Rollerfson I (2005) Indigenous breeds, local communities. documenting animal breeds and breeding from a community perspective. GTZ, FAO, LIFE Initiative. Sadri, Rajasthan, India. 66p.

SIAP (2020) Población ganadera. Inventario 2019 Caprino. Servicio de Información Agroalimentaria y Pesquera. https://www.gob.mx/cms/uploads/attachment/file/564339/Inventario_2019_caprino.pdf Fecha de consulta: 5 de enero de 2021.

Sierra A, Molina A, Delgado J, Hernández J, Rivera M (1997) Zootechnical description of the creole goat of the Oaxaca region (Mexico). Animal Genetic Resources Information 21: 61-70.

Soriano RR, Arias MA, Armella VM, Yañez LL, Almaraz BI (2017) Identificación de atributos de sustentabilidad en una producción caprina en la Mixteca baja usando análisis multivariado. En: Huerta PA, Díaz RR (ed). Agricultura sostenible una transición desde lo tradicional. México. pp: 285-295.

Torres-Acosta JF, Villarroel-Álvarez MS, Rodríguez-Arévalo F, Gutiérrez-Segura I, Alonso-Díaz MA (2003) Diagnóstico de nematodos gastrointestinales resistentes a bencimidazoles e imidazotiazoles en un rebaño caprino de Yucatán, México. Revista Biomédica 14: 75-81. 
Tórtora JL (1987) Ectima contagioso de ovinos y caprinos. Ciencia Veterinaria 4: 257-283.

Trujillo Ortiz CX, Vargas López S, Torres Hernández G, Bustamante González Á, Becerril Pérez CM, Guerrero Rodríguez JD (2010) Análisis del proceso de introducción de cabras exóticas en los sistemas campesinos de la Mixteca poblana. En: Los grandes retos para la ganadería: hambre, pobreza y crisis ambiental. Chapingo, México. pp: 425-433.

Valerio D, García A, Perea J, Acero R y Gómez G (2009) Caracterización social y comercial de los sistemas ovinos y caprinos de la región noreste de república Dominicana. Interciencia 34: 337-3644

Varese S, Escárcega S (2004) La ruta Mixteca. El impacto etnopolítico de la migración en los pueblos indígenas de México. Primera edición. Colección pluralidad cultural en México. UNAM. México. 422p.

Villarreal-Arellano HR, Fuentes-Mascorro G, Ramírez-Bribiesca JE, Torres-Hernández G, Ricardi DCC, VargasLópez S (2020) Morphostructural variability in the pastoreña goat in different regions of the Mixteca of México: A phenotypic study to establish the racial profile. Revista de La Facultad de Ciencias Agrarias 52: 360-375.

Zepeda JSH, Guerra FJF, García MH, Serrano ER, Vázquez ACS, Cruz AB, Bermejo JVD (2002) Estudios de los recursos genéticos de México: características morfológicas y morfoestructurales de los caprinos nativos de Puebla. Archivos de Zootecnia 51: 53-64. 